\title{
Annotation
}

\section{Treating glucosphingolipid disorders by chemotherapy: Use of approved drugs and over-the-counter remedies*}

\author{
N. S. RADIN \\ Mental Health Research Institute, The University of Michigan, Ann Arbor, \\ Michigan, USA. \\ Correspondence: 350 Sharon Park Dr., Apt. S5, Menlo Park, CA 94025, USA. \\ E-mail: Glyconorm@aol.com
}

MS received 25.04.00 Accepted 3.07.00

\begin{abstract}
Summary: The accumulation of a glucosphingolipid (GSL) in individuals lacking an adequate level of hydrolase activity could be minimized by chemotherapeutic measures that slow the formation of the GSL and stimulate the defective hydrolase. By achieving a balance in the rates of formation and breakdown, one should be able to alleviate the symptoms of excess storage and achieve a satisfactory accommodation. While several drugs seem to be specifically suitable for this purpose, only one of these has been approved for human use. However, less effective drugs and over-the-counter substances are available for human use and may prove satisfactory for a few years until better ones are made available. The proposed materials and the evidence behind the recommendations are presented in this paper.
\end{abstract}

Disclaimer: The ideas expressed here are strictly my own and the Journal of Inherited Metabolic Disease certainly does not endorse the proposals. I am not a medical doctor, merely a biochemist with 48 years of research experience with sphingolipids, and must point out to the medical and lay (patient) reader that none of the approaches have been tried for the purpose of sphingolipidosis therapy. The approaches are based on published work in peer-edited journals, referenced below so that the reader can check the accuracy of the citations and the quality of the research work. Many — not all —of the reported obser-

* The views expressed in this article are those of the author and not necessarily those of the Journal. They are intended to provide a forum to stimulate debate on concepts for treatment, but in a controlled manner. 
vations were obtained with cultured cancer cells, which are quite different from live individuals, despite the claims of some animal protectionists. The drugs and 'health supplements' named here are in common use and deserve serious consideration. Probably the best way to proceed is to start with one approach, then add a second, then add a third. As with all pharmaceutical products, combinations of the suggested materials might interact in unexpected ways. An open-minded, caring doctor must approve the suggestions and intelligently monitor the results of their use. Each patient must decide for himself or herself whether the unknown risks inherent in any form of therapy are warranted by the intensity of the affliction.

The dosages used with the currently approved drugs were all designed to achieve a specific therapeutic effect, usually the death of cancer cells. (There is an important clue in this relationship: cancer of the B cells is especially common in Gaucher patients and elevated levels of glucosylceramide (GlcCer) produce cell proliferation (Radin 1999).) I doubt that anyone analysed a cancer patient for his GlcCer content during chemotherapy, so one cannot guess whether the drug would indeed help a glucosphingolipidosis patient. Only the standard empirical method-gradual increases in dosage - can tell us the optimum dose for metabolite balance therapy. Perhaps a research-minded researcher will run appropriate trials after reading this article. This is important, because the treatments will have to be continued until more specific and reactive drugs are approved for human use-perhaps until the end of 2002. A patient following my suggestions should not expect rapid recovery to a normal state because of the abnormally low activities of the defective enzyme and the large 'reservoir' of accumulated GSL. Once the stored GSL has been brought to the normal level, therapy can be stopped for a while, or reduced in intensity, since the GSL accumulation process is usually rather slow.

My suggestions should also be considered with the idea in mind of simultaneously using commercially available enzymes, such as Glucerase or Ceredase, but at a lower dosage. This would greatly reduce the cost of therapy. Similarly, the suggested treatments could be used to reduce the dosage of a GlcCer synthase inhibitor once it has been approved for use.

\section{THE RATIONALE}

The glucosphingolipid disorders (Gaucher, Tay-Sachs, Sandhoff, Fabry, GM1gangliosidosis, fucosidosis) are genetic disorders due to abnormally low activities of the hydrolases (or their natural stimulators) that break down glucosphingolipids (GSLs) to smaller molecules. As with most chemicals in the body, the GSLs are formed by cells; then, after they have 'done their duty' for a certain length of time, they are broken down into smaller molecules that can be recycled to make new GSLs or broken down further for other uses. In patients with these genetic disorders, the ability to hydrolyse a specific GSL is low and the unremitting flow of new GSL 
molecules produces a pile-up of accumulating GSLs that cannot carry out their function and simply cause trouble.

In Gaucher disease, there is accumulation of the simplest GSL, GlcCer. The defective enzyme is GlcCer glucosidase, which normally removes glucose from GlcCer, leaving behind a basic sphingolipid, ceramide. The other GSLs in this disease group are all formed from GlcCer by enzymes that attach additional sugars and other moieties. In sulphatidosis, several GSLs accumulate, most of them derived from GlcCer, and the techniques described in this paper might alleviate some of the symptoms in this disorder. The same applies to Fabry patients.

At present it is possible to periodically inject Gaucher patients with GlcCer glucosidase (modified to improve cellular uptake) and thus overcome the lack of adequate enzyme activity. The cost of this enzyme is enormous. Fabry patients may soon be able to supplement their defective $\alpha$-galactosidase with injections of normal enzyme, also at great financial cost. Some day it may be possible to get injections of the normal gene that will once-and-for-all displace the defective gene and produce a long-lived cure. Since 1981 I have been publishing articles proposing a simpler treatment for the sphingolipid disorders: slow the rate of synthesizing the GSL that has been accumulating, so that the rate of formation equals the patient's rate of GSL breakdown (Radin 1996). Simultaneously, I suggest in this paper, one should also stimulate the rate of GSL breakdown. I suggest calling this approach metabolite balance therapy. Several methods could be used, together or separately, to achieve the balance: (a) the enzyme that produces the undesired metabolite could be slowed by an inhibitor; or (b) the enzyme's activator (if known) could be prevented from binding to the enzyme; or (c) the concentration of substrate used by the enzyme could be reduced by slowing its formation or diverting it to a different metabolic pathway; or (d) the speed of the metabolite's destruction could be increased by stimulating a suitable enzyme. Other methods could also be useful. Metabolite balance therapy need not be restricted to the GSL disorders.

This idea is based on the fact that many sphingolipidosis patients contain some residual hydrolytic activity, sometimes as much as $10-30 \%$ of the normal amount of activity. The rate of formation need not be slowed much, since the normal activity of the hydrolase is somewhat greater than the normal activity of the synthesizing enzyme. In other words, the normal body has excess degradative activity compared to the amount of synthase and can accommodate to appreciable loss of the degradative enzyme. My proposal is thus based on simple arithmetic and it assumes that the slowed rate of synthesis and accelerated rate of hydrolysis will not cause damage to the patient and that the accumulated lipid is accessible to the defective hydrolase. Ideally, normalizing the tissue concentration of the affected GSL will not result in accumulation of precursors or decreases in the GSLs derived from the affected GSL. It is possible that more than one enzyme will have to be controlled.

Other researchers have called this approach 'substrate deprivation therapy' (Abe et al 2000b; Jeyakumar et al 1999). This term would be appropriate if, indeed, a substrate for the enzyme to be slowed is actually reduced in concentration, as in approach (c). In the case of Gaucher disease, the synthase's substrates are ceramide and UDP-glucose, not GlcCer. Reducing the concentration of either substrate would 
slow the synthesis of GlcCer. If one simply inhibits the enzyme, the term is inappropriate. For patients suffering disorders of the more complex GSLs that accumulate in the (non-Gaucher) glucosphingolipid storage diseases, lowering the concentration of GlcCer might slow the enzymes involved in the synthesis of the undesired metabolite and 'substrate deprivation' would be an appropriate term. The essence of the balance approach, however, is in equalizing the rates of anabolism and catabolism.

\section{PROOF OF CONCEPT}

Several recent reports support the value of metabolite balance therapy. One study was done with mice suffering from a galactosphingolipidosis, globoid cell leukodystrophy (LeVine et al 2000). These 'twitcher' mice have an inadequate activity of the $\beta$-galactosidase acting on galactosylceramide. Slowing the synthesis of the galactolipid by slowing the synthesis of one of its precursors, 'ketosphingosine' (2-amino-3-keto-octadecanol-1), with L-cycloserine produced a partial beneficial effect on the mice. Since cycloserine blocks formation of all the sphingolipids, this technique cannot produce a fully satisfactory effect, but it might have merit when combined with a more specific method of achieving metabolite balance. It was found to augment the depleting action of an inhibitor of GlcCer synthesis, PDMP (see below), producing a $50 \%$ loss of GlcCer and 33\% loss of lactosylceramide in mouse kidneys after 4 days (Shukla et al 1991). Note that the cycloserine that is used as an antibiotic is the D-isomer.

A similar test utilized oral administration of NB-DNJ ( $N$-butyldeoxynojirimycin) to slow GlcCer synthase in human subjects suffering with Gaucher disease (Cox et al 2000). This inhibitor is not as potent or as specific as one would desire, since it also inhibits a glycoprotein $\alpha$-glucosidase, but it was tolerated over a year, diarrhoea being the major side-effect (Cox et al 2000; Platt and Butters 1998). Peripheral neuropathy appeared in two of the 28 subjects. A sign of a positive effect was a decrease of $39 \%$ within a year in ganglioside GM1, a product of GlcCer, measured in white cells. Other signs of effectiveness were decreases of $7 \%$ and $15 \%$ in liver and spleen sizes, respectively. Haemoglobin and platelet concentrations also improved. A chemically similar, more specific inhibitor has been found to show fewer side-effects in animals but has not yet been approved for human use (Andersson et al 2000).

Another recent test of metabolite balance therapy was described for knockout mice lacking the $\alpha$-galactosidase needed for globotriaosylceramide (Gb3) hydrolysis (Abe et al 2000b). This is a form of Fabry disease that is extreme, in that the enzyme concentration is zero. Thus one can only show that the rate of GSL accumulation is lower than in untreated mice. Here a new variant of D-threo-PDMP was used to block GlcGer synthesis. PDMP is D-threo-1-phenyl-2-decanoylamino-3morpholino-1-propanol; PPPP ('P4') is 1-phenyl-2-palmitoylamino-3-pyrrolidino1-propanol; and the variant was $3^{\prime}, 4^{\prime}$-ethylenedioxy-P4 (Lee et al 1999). All three drugs strongly inhibit the synthesis of GlcCer. PDMP has also been shown to slow lactosylceramide and Gb3 synthesis (Chatterjee et al 1996). Eight-week-old mice 
that had already accumulated a significant amount of Gb3 were given intraperitoneal injections twice a day with the 'P-drug' at $10 \mathrm{mg} / \mathrm{kg}$ for 8 weeks. The concentrations of $\mathrm{Gb} 3$ found in kidney, liver and heart were roughly one-half that seen in the control mice. No signs of toxicity were seen when the treated mice were compared with regard to weight of body and six different organs, blood urea nitrogen and other markers. Electron microscopy showed the usual Fabry lipid inclusions in the control mice, but these were absent from the treated mice. Thus this test demonstrates the safety and effectiveness of the approach, even in an extreme form of a sphingolipidosis.

The same P-drug was also shown to normalize cultured Fabry cells that had been transformed with the Epstein-Barr virus (Abe et al 2000a). The method used to monitor the decrease in $\mathrm{Gb} 3$, measurement of the amount of fluorescent verotoxin binding to the cells, could be used to monitor treated patients.

\section{THE TOXICITY QUESTION}

In a study with normal mice, PDMP was found to quickly lower the concentration of GlcCer in the kidneys without producing any noticeable harm (Shukla et al 1991). The decrease was one-third of the normal concentration after only $5 \mathrm{~h}$. Young mice treated with high doses of PDMP for 12 days gained less weight than control mice and, $40 \mathrm{~h}$ later, were found to have relatively normal organ weights except for the kidneys, which were 11\% smaller (Inokuchi et al 1987). Blood cell counts were normal. Other mice that were injected with cancer cells and then treated with PDMP for 10 days survived the cancer exposure and lived as active a life as the controls for months.

A study with normal mice and NB-DNJ over a 118-day period also yielded large decreases in several GSLs in several organs (Platt et al 1997). Mice treated for 8 weeks with NB-DNJ looked and grew normally, but the spleen and thymus developed cell loss. Nevertheless, their immune system seemed to be normal. These studies showed no marked toxicity due to the inhibitors. A lower dose could probably give fewer side-effects yet be effective for simply slowing GSL synthesis. As noted above, Gaucher patients improved on treatment with NB-DNJ ( $\sim 300 \mathrm{mg} /$ day) for over a year despite problems with diarrhoea. Mice treated with P-drugs did not exhibit diarrhoea, possibly because of higher specificity of action.

The GSLs, which take part in many cell functions and which cause so much anguish when they accumulate, do not seem to be very essential for life, at least in the laboratory. Yeast cells that are unable to make sphingolipids because of a genetic error grow almost as fast as normal yeast. Fish raised from the embryonic stage in water containing PDMP, which greatly depleted them of their GSLs, grew almost as fast as fish in plain water during the hatching phase (Fenderson et al 1992). After they were transferred to plain water, they could not be distinguished from the control fish. Cells growing in the presence of a P-drug lose almost all their GSLs but survive at least a week, provided the concentration of inhibitor is not too high. Perhaps most of the GSL in normal people is like stored fat, used as a backup 
for stresses or emergencies. It is also possible that a chronic deficiency of GSLs has important beneficial value (Radin 1999).

The rest of this article describes the available materials that can be expected to deplete patients of their accumulated GSLs.

\section{SLOWING GIcCer SYNTHESIS}

From Myles Cabot's laboratory we learn that tamoxifen, verapamil, and doxorubicin slow GlcCer synthesis from UDP-glucose and ceramide (Cabot et al 1996; Lavie et al 1997). Doxorubicin (Adriamycin) tends to be somewhat toxic and it normally has to be injected slowly into patients over a long period, so it does not appear suitable for chronic use. Nevertheless, it might be useful in crisis situations or as an initial treatment. Tamoxifen can be used chronically; it finds much use in breast cancer therapy and looks very promising for the prevention of breast cancer metastasis and even for preventing breast cancer in the first place.

The abortion-inducing drug, RU486 (mifepristone), inhibits conversion of ceramide to GlcCer (Lucci et al 1999) and has also shown promise in cancer therapy (Koide 1998). It is especially effective in vivo when combined with a second inhibitor of GlcCer synthase, tamoxifen (El Etreby and Liang 1998). This drug has been much studied in normal and cancer-bearing women and might be very valuable in glucosphingolipidosis therapy. Relatively long-term tests indicate that it produces slight to moderate adverse effect, attributed to its inhibition of glucocorticoids (Koide 1998). While anti-abortionists have fought against its use, it is available in several countries and will surely soon be available in the United States.

A vitamin A derivative showing promise in cancer therapy, all-trans-retinoic acid, was found to slow the formation of GlcCer (Madison et al 1998). A related drug made from retinoic acid, fenretinide, may be a more effective inhibitor (Maurer 1999) and has been tried in patients.

There are probably other antineoplastic drugs that also act against GlcCer synthesis but simply have not been tested for that capability. A test of this hypothesis might prove very useful.

Several reports have shown that concentrated glucose in growth media promotes cell growth (El-Khatib et al 1996). The level of UDP-glucose is increased by a high glucose level in vivo, and this nucleotide has been shown to be rate-limiting in the synthesis of GlcCer in kidney cells (Zador et al 1993). Diabetic rats developed enlarged kidneys owing to the high blood glucose levels, and lowering GlcCer levels with PDMP shrank the kidneys back to normal despite the high glucose availability. Since GlcCer promotes cell growth (Radin 1999), the growth-promoting property of glucose seems to be expressed by more rapid GlcCer synthesis. Conversely, one finds slow growth in low-glucose media; thus one would expect to get slower GSL synthesis if the glucose level in tissues were below normal. Because the brain needs so much glucose, we cannot let our blood glucose concentration decrease very far (perhaps $70 \mathrm{mg} / 100 \mathrm{ml}$ is safe), but it is certainly possible to reduce one's sugar intake appreciably, given sufficient incentive. Some diabetics are greatly helped by intake of a drug (such as acarbose or miglitol) that slows the digestion of starches, 
so that blood glucose levels do not rise as much as usual after a meal. Perhaps such drugs would help to slow postprandial elevation of GSL synthesis in nondiabetic sphingolipidosis patients.

Glucose is formed in the body from dietary carbohydrates and amino acids, so a reduction in total food intake can be expected to slow the synthesis of GSLs. Studies with various animal species have clearly demonstrated that a calorie-restricted diet leads to considerable prolongation of life and postponement of degenerative disorders. Part of the explanation may devolve on the reduction in plasma insulin-like growth factor-1 seen in such animals (Sonntag et al 1999). This factor acts to elevate plasma glucose levels, so a reduction in IGF-1 can be expected to slow GlcCer synthesis.

Another way to slow the conversion of glucose to GlcCer is to eat sugars that compete against glucose for the enzymes involved in GlcCer synthesis. Glucosamine, a naturally occurring sugar, is readily available as an over-the-counter tablet for treatment of joint pain. Its ability to extend the survival of mice bearing Ehrlich ascites carcinoma (Gonzalez and Amos 1977) suggests that it does slow GlcCer synthesis in vivo (Radin 1999). This observation is consistent with the similar effect of PDMP (Inokuchi et al 1987). Tests with rats, using quite high doses of glucosamine, have yielded undesirable changes in the way the body uses glucose and insulin, but there is probably no harm in somewhat lower dosages, as recommended on the bottle labels. Human studies have used over $1.5 \mathrm{~g} /$ day, apparently with minor effects, but long-term effects have not been studied.

Glucocorticoid-like drugs used widely for various purposes should be avoided, if possible, since they stimulate the synthesis of ceramide and GSLs (Cifone et al 1999; Levi et al 1995). Dexamethasone and prednisone are in this category.

Chlorpromazine, a psychoactive drug having quieting effects, was found to lower GlcCer levels in mice considerably (Hospattankar et al 1982). The mechanism of the effect was not studied. Obviously it should be used at a low dosage.

\section{SLOWING GSL SYNTHESIS BY LOWERING THE AVAILABILITY OF CERAMIDE}

Since the GSLs are formed from ceramide by adding sugar molecules, lowering the availability of ceramide should slow their synthesis. Broken cell preparations used for assaying GlcCer synthase show increased synthesis if ceramide is added to the incubation tube, suggesting that the enzyme is normally unsaturated with regard to ceramide availability. Some of the ceramide is derived from a phosphosphingolipid, sphingomyelin, by several hydrolases. Thus one should minimize the rate of sphingomyelin breakdown. One factor that speeds the enzymatic breakdown is arachidonic acid (Surette et al 1999), a polyunsaturated fatty acid found in vegetable oils and animal fats, which should be avoided. Olive oil and canola oil have a relatively low content of arachidonic acid and are therefore to be preferred. The risk in eating corn oil is illustrated by the observation that injection of the oil intraperitoneally into mice produced an elevation in tissue GlcCer (Abe et al 2000b). 
Commercially prepared foods lacking fat are increasingly becoming available but some of them include a high sugar content.

Eating fat is undesirable in another way, since part of dietary fat (and carbohydrates) is converted in the body to palmitic acid, the precursor of sphingosine. The palmitate ends up in both the amine and fatty acid moieties of ceramide. Adding palmitic acid to cultured heart muscle cells produced ceramide accumulation (Hickson-Bick et al 2000).

Another way to slow the formation of ceramide from sphingomyelin is by taking steps to increase the concentration of glutathione (GSH) in the body. GSH normally slows the hydrolysis of sphingomyelin (Liu and Hannun 1997; Liu et al 1998) and the body adjusts its concentration as needed. It is destroyed by oxygen radicals, which are formed by normal processes involving reactions between body constituents and oxygen, as well as by radiation from natural radioactive elements in one's body. These are the free radicals that produce cell damage and speed the ageing processes. Food antioxidants, such as vitamin E, act to slow ceramide formation from sphingomyelin by protecting GSH from destruction by oxygen, and thus slow GlcCer formation. This suggests that it would be helpful to supplement one's diet with antioxidants. GSH is available from nutrition stores. Other dietary components have been recommended for this purpose: tofu, broccoli, cauliflower, and Brussels sprouts. There is a very large amount of literature on GSH and on antioxidant foods, so it pays to study it in order to match one's food preferences and my dietary recommendation.

GSH is normally in equilibrium with its oxidized form, GSSG, and eating thiols will push the equilibrium in the direction of more GSH. $N$-Acetylcysteine seems to be a suitable thiol.

Carnitine, another important body component sold as a nutritional supplement, has been shown to lower cellular levels of ceramide (Andrieu-Abadie et al 1999). It is an important component of the body's fat-burning apparatus and it ought to be helpful, especially since it aids in the oxidative destruction of palmitic acid, the precursor of the sphingolipids.

The use of marijuana ought to be avoided as its primary component, $\Delta^{9}$ tetrahydrocannabinol, speeds the formation of ceramide from sphingomyelin (Galve-Roperh et al 2000). Similarly, the use of dietary arginine supplementation (to aid blood flow) should be avoided, since arginine acts to stimulate nitric oxide production, which in turn stimulates destruction of GSH and conversion of sphingomyelin to ceramide (Ma et al 1999).

As mentioned earlier, L-cycloserine slows the synthesis of ceramide in vivo. Many researchers have used fumonisin $\mathrm{B} 1$ in vitro to slow ceramide synthesis (via acylation of sphingosine), but it tends to produce cancer, probably because it diverts sphingosine into toxic metabolic products.

\section{SPEEDING THE BREAKDOWN OF GlcCer}

This can be done by injecting phosphatidylserine periodically, since it stimulates activity of $\beta$-glucosidase, the enzyme that removes the glucose portion of GlcCer. 
The effect was shown in mice containing a high content of GlcCer ('Gaucher mice') (Datta and Radin 1988). This lipid also stimulates neutral/alkaline ceramidase (El Bawab et al 1999), so it would exert a second beneficial effect by lowering the availability of ceramide (see above). It was also found to stimulate the hydrolysis of lactosylceramide and ganglioside GM1, even in cells from patients with Krabbe disease and GM1 gangliosidosis (Kobayashi et al 1985).

The phospholipid is available from 'nutrition' stores, which tout it as a memory enhancer for elderly people, to be taken orally. It seems unlikely that it would survive passage intact through the digestive system, but perhaps a patient would absorb a useful portion of the material. In view of the high price of this lipid to scientists, I would want reliable assurance as to the purity of the preparation. No doubt better stimulators of the sphingolipid hydrolases could be synthesized.

A complication in the use of phosphatidylserine is that it stimulates neutral sphingomyelinase to produce ceramide, which might neutralize the effort to lower tissue ceramide.

\section{HORMONAL CONTROL OVER GICCE SYNTHESIS}

Mouse kidney was found to be sensitive to androgen and oestrogen levels (Shukla et al 1992). Young mice injected with testosterone showed an elevated level of GlcCer synthase activity, more rapid synthesis of GSLs, and faster growth. Moreover the activity of GlcCer glucosidase was decreased, an effect that joins in with the first effect to enhance GSL synthesis. 17 $\beta$-Oestradiol exerted opposite effects on GlcCer synthase and hydrolase, slowing growth or shrinking the kidneys. It seems likely that other hormone-sensitive tissues would respond similarly. The prostate gland is certainly one of those. Thus glucosphingolipidoses in those sites should respond to anti-androgen, pro-oestrogen therapy by lowering GlcCer and other GSL production. Anti-androgen drugs such as Lupron (long-lasting injectable) and casodex should be useful. These drugs are used for long periods in men with prostate cancer, and should be useful at lower doses without significant sexual impairment in men. Oestrogen-like drugs, also at low doses, should help. It is interesting that tamoxifen has oestrogenic activity and also inhibits GlcCer synthesis, as mentioned above. This makes its use more attractive.

\section{REFERENCES}

Abe A, Arend LJ, Lee L, Lingwood C, Brady RO, Shayman JA (2000a) Glycosphingolipid depletion in Fabry disease lymphoblasts with potent inhibitors of glucosylceramide synthase. Kidney Int 57: 446-454.

Abe A, Gregory S, Lee L, et al (2000b) Reduction of globotriaosylceramide in Fabry disease mice by substrate deprivation. J Clin Invest 105: 1563-1571.

Andersson U, Butters TD, Dwek RA, Platt FM (2000) $N$-Butyldeoxygalactonojirimycin: a more selective inhibitor of GSL biosynthesis than $N$-butyldeoxynojirimycin, in vitro and in vivo. Biochem Pharmacol 59: 821-829. 
Andrieu-Abadie N, Jaffrézou JP, Hatem S, Laurent G, Levade T, Mercadier JJ (1999) L-Carnitine prevents doxorubicin-induced apoptosis of cardiac myocytes: role of inhibition of ceramide generation. FASEB $J$ 13: 1501-1510.

Cabot MC, Giuliano AE, Volner A, Han TY (1996) Tamoxifen retards glycosphingolipid metabolism in human cancer cells. FEBS Lett 394: 129-131.

Chatterjee S, Cleveland T, Shi WY, Inokuchi J, Radin NS (1996) Studies of the action of ceramide-like substances (D- and L-threo-PDMP) on sphingolipid glycosyltransferases and purified lactosylceramide synthase. Glycoconjugate $J$ 13: 481-486.

Cifone MG, Migliorati G, Parroni R, et al (1999) Dexamethasone-induced thymocyte apoptosis: apoptotic signal involves the sequential activation of phosphoinositide-specific phospholipase C, acidic sphingomyelinase, and caspases. Blood 93: 2282-2296.

Cox T, Lachmann R, Hollak C, et al (2000) Novel oral treatment of Gaucher's disease with $N$-butyldeoxynojirimycin (OGT 918) to decrease substrate biosynthesis. Lancet 353: 1481-1485.

Datta SC, Radin NS (1988) Normalization of liver glucosylceramide levels in the "Gaucher" mouse by phosphatidylserine injection. Biochem Biophys Res Commun 152: 155-160.

El Bawab S, Bielawska A, Hannun YA (1999) Purification and characterization of a membrane-bound nonlysosomal ceramidase from rat brain. J Biol Chem 274: 27948-27955.

El Etreby MF, Liang Y (1998) Effect of antiprogestins and tamoxifen on growth inhibition of MCF-7 human breast cancer cells in nude mice. Breast Cancer Res Treat 49: 109-117.

El-Khatib M, Radin NS, Shayman JA (1996) Glycosphingolipid synthesis and proliferation in a renal cell line grown in high glucose. Am J Physiol 270 (Renal Fluid Electrolyte Physiol 39): F476-F484.

Fenderson BA, Ostrander GK, Hausken Z, Radin NS, Hakomori S (1992) A ceramide analog (PDMP) inhibits glycolipid synthesis in fish embryos. Exp Cell Res 198: 362-366.

Galve-Roperh I, Sanchez C, Cortes ML, del Pulgar TG, Izquierdo M, Guzman M (2000) Anti-tumoral action of cannabinoids: involvement of sustained ceramide accumulation and extracellular signal-regulated kinase activation. Nature Medicine 6: 313-319.

Gonzalez F, Amos H (1977) Effects of naturally occurring sugars on Ehrlich ascites tumor growth in mice. J Natl Cancer Inst 58: 1519-1522.

Hickson-Bick DLM, Buja LM, McMillin JB (2000) Palmitate-mediated alterations in the fatty acid metabolism of rat neonatal cardiac myocytes. J Mol Cell Cardiol 32: 511-519.

Hospattankar AV, Vunnam RR, Radin NS (1982) Changes in liver lipids after administration of 2-decanoylamino-3-morpholinopropiophenone and chlorpromazine. Lipids 17: 538-543.

Inokuchi J, Mason I, Radin NS (1987) Antitumor activity in mice of an inhibitor of glycosphingolipid biosynthesis. Cancer Lett 38: 23-30.

Jeyakumar M, Butters TD, Cortina-Borja M, et al (1999) Delayed symptom onset and increased life expectancy in Sandhoff disease mice treated with $N$-butyldeoxynojirimycin. Proc Natl Acad Sci USA 96: 6388-6393.

Kobayashi T, Shinnoh N, Goto I, et al (1985) Galactosylceramide- and lactosylceramide-loading studies in cultured fibroblasts from normal individuals and patients with globoid cell leukodystrophy (Krabbe's disease) and GM1-gangliosidosis. Biochim Biophys Acta 835: 456-464.

Koide SS (1998) Mifepristone-auxiliary therapeutic use in cancer and related disorders. $J$ Reprod Med 43: 551-560.

Lavie Y, Cao H, Volner A, et al (1977) Agents that reverse multidrug resistance, tamoxifen, verapamil, and cyclosporin A, block glycosphingolipid metabolism by inhibiting ceramide glycosylation in human cancer cells. J Biol Chem 272: 1682-1687. 
Lee L, Abe A, Shayman JS (1999) Improved inhibitors of glucosylceramide synthase. J Biol Chem 274: 14662-14669.

Levi M, Shayman JA, Abe A, et al (1995) Dexamethasone modulates rat renal brush border membrane phosphate transporter mRNA and protein abundance and GSL composition. $J$ Clin Invest 96: 207-216.

LeVine SM, Pedchenko TV, Bronshteyn IG, Pinson DM (2000) L-Cycloserine slows the clinical and pathological course in mice with globoid cell leukodystrophy (twitcher mice). $J$ Neurosci Res 60: 231-236.

Liu B, Hannun YA (1997) Inhibition of the neutral magnesium-dependent sphingomyelinase by glutathione. $J$ Biol Chem 272: 16281-16287.

Liu B, Andrieu-Abadie N, Levade T, Zhang P, Obeid LM, Hannun YA (1988) Glutathione regulation of neutral sphingomyelinase in tumor necrosis factor-alpha-induced cell death. $J$ Biol Chem 273: 11313-11320.

Lucci A, Han TY, Liu YY, Giuliano AE, Cabot MC (1999) Modification of ceramide metabolism increases cancer cell sensitivity to cytotoxics. Int J Oncol 15: 541-546.

Ma Q, Williamson KE, O'Rourke D, Rowlands BJ (1999) The effects of L-arginine on crypt cell hyperproliferation in colorectal cancer. J Surg Res 81: 181-188.

Madison KC, Sando GN, Howard EJ, et al (1998) Lamellar granule biogenesis: a role for ceramide glucosyltransferase, lysosomal enzyme transport, and the Golgi. J Invest Dermatol Symp Proc 3: 80-86.

Maurer BJ, Metelitsa LS, Seeger RC, Cabot MC, Reynolds CP (1999) Increase of ceramide and induction of mixed apoptosis necrosis by $N$-(4-hydroxyphenyl)-retinamide in neuroblastoma cell lines. J Natl Cancer Inst 91: 1138-1146.

Platt FM, Butters TD (1998) New therapeutic prospects for the glycosphingolipid lysosomal storage diseases. Biochem Pharmacol 56: 421-430.

Platt FM, Neises GR, Reinkensmeier G, et al (1997) Prevention of lysosomal storage in Tay-Sachs mice treated with $N$-butyldeoxynojirimycin. Science 276: 428-431.

Radin NS (1996) Treatment of Gaucher's disease with an enzyme inhibitor. Glycoconjugate J 13: $153-157$.

Radin NS (1999) Chemotherapy by slowing glucosphingolipid synthesis. Biochem Pharmacol 57: 589-595.

Shukla A, Shukla GS, Radin NS (1992) Control of kidney size by sex hormones; possible involvement of glucosylceramide. Am J Physiol 262: F24-F29.

Shukla G, Shukla A, Inokuchi J, Radin NS (1991) Rapid kidney changes resulting from glycosphingolipid depletion by treatment with a glucosyltransferase inhibitor. Biochim Biophys Acta 1083: 101-108.

Sonntag WE, Lynch CD, Cefalu WT, et al (1999) Pleiotropic effects of growth hormone and insulin-like growth factor IGF-1 on biological aging: inferences from moderate caloric-restricted animals. J Gerontol A Biol Sci Med Sci 54: B521-B538.

Surette ME, Fonteh AN, Bernatchez C, Chilton FH (1999) Perturbations in the control of cellular arachidonic acid levels block cell growth and induce apoptosis in HL-60 cells. Carcinogenesis 20: 757-763.

Zador IZ, Deshmukh GD, Kunkel R, Johnson K, Radin NS, Shayman JA (1993) A role for glycosphingolipid accumulation in the renal hypertrophy of streptozotocin-induced diabetes mellitus. J Clin Invest 91: 797-803. 